\title{
A Review of Pattern Languages for Software Documentation
}

\author{
João Santos \\ up201809187@fe.up.pt \\ Faculty of Engineering, \\ University of Porto \\ Porto, Portugal
}

\author{
Filipe Figueiredo Correia \\ filipe.correia@fe.up.pt \\ Faculty of Engineering, \\ University of Porto \\ INESC TEC \\ Porto, Portugal
}

\begin{abstract}
Software documentation is an important part of the captured knowledge of a software project and documentation patterns have often been used as a systematic way to describe good practices on software documentation. Still, many software teams are challenged by what to document, how to keep the documentation consistent and how to make their consumers aware of the relevant documents. A literature review was done over 14 publications and identified 16 quality attributes and 114 patterns about software documentation. This knowledge was analysed and classified and led to the proposal of new categories and relationships between the existing patterns. These are depicted as a new pattern map that provides a new perspective of documentation patterns and can be used to guide teams in adopting software documentation practices.
\end{abstract}

\section{CCS CONCEPTS}

- Software and its engineering $\rightarrow$ Software creation and management.

\section{KEYWORDS}

software documentation, documentation patterns, documentation issues, quality attributes, documentation engineering

\section{ACM Reference Format:}

João Santos and Filipe Figueiredo Correia. 2020. A Review of Pattern Languages for Software Documentation . In European Conference on Pattern Languages of Programs2020 (EuroPLoP '20), Fuly 1-4, 2020, Virtual Event, Germany. ACM, New York, NY, USA, 14 pages. https://doi.org/10.1145/3424771.3424786

\section{INTRODUCTION}

Documentation engineering-the set of procedures needed to write and maintain artifacts of documentation-is often neglected in software projects, and it is considered tedious and time-consuming by many [13]. Notwithstanding, software documentation can be an important part of the captured knowledge of a software project because it is a way to record non-structured and informal content and it's been considered a recommended practice in software development and maintenance since its early days [14].

One goal of documentation is to help developers, testers, managers and end-users to understand their systems and to collaborate.

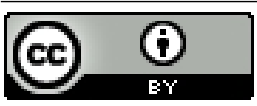

This work is licensed under a Creative Commons Attribution International 4.0 License. EuroPLoP '20, July 1-4, 2020, Virtual Event, Germany

(C) 2020 Copyright held by the owner/author(s)

ACM ISBN 978-1-4503-7769-0/20/07.

https://doi.org/10.1145/3424771.3424786
It facilitates communication and can be expressed in different forms, such as wikis, code annotations, user manuals and system requirements [2].

Even when teams see it as important, projects often lack consistent, useful or even up-to-date documentation [15]. This is, to a large extent, due to the difficulty of writing and maintaining it [9].

This work is guided by two research questions, that stem from the observation that there is a significant amount of information regarding software documentation practices, but that it is dispersed and, as such, doesn't efficiently guide developers in the process of adopting them. This can be the reason for a lack of awareness of the benefits that may be gained from using these practices, and of how to use them given the specific context of each team and project.

- RQ1: What quality attributes are important when creating and using software documentation?

- RQ2: What are the best practices for documenting software?

To generate insights and possible answers to these questions we conduct a literature review and a comprehensive study of documentation in published works, including specifically what are the main quality attributes of documentation artifacts and what documentation best practices have been documented as patterns. Often in the software engineering field, best practices are defined as pattern languages $[5,16,17]$ which are a way to characterize recurrent problems and their solutions in the contexts in which they appear, and to combine them within a certain domain [3]. The data gathered in this study systematizes knowledge on the patterns that are currently known, and on how they relate, using proven pattern mining techniques [11] and proposing new categories and relationships between the existing patterns. The main goal of this work is not to document new patterns but to map out the existing patterns and pattern catalogs, find relationships that hadn't yet been identified in the literature, and hopefully facilitate navigating the pattern languages and help software development teams adopt these practices.

\section{QUALITY ATTRIBUTES}

We have first surveyed the literature for documentation issues described in existing publications [1, 2, 4, 6-10, 12, 13, 15, 18-20], and cataloged a comprehensive list of attributes that should be taken in consideration when writing or maintaining documentation.

These quality attributes are also used in this work to relate and group existing patterns, which can to guide a team seeking to achieve them. 


\subsection{Methodology}

We surveyed and analysed the literature for documentation issues following these steps:

(1) Collect publications that describe some kind of issue on software documentation including those that describe documentation patterns. In particular, we searched for literature using Google Scholar using keywords related to software documentation, such as "patterns software documentation", "software documentation", "software documentation issues" and "software documentation practices". The relevant literature was chosen based on title and abstract;

(2) Remove duplicates in the dataset, as we have found the same issues covered in different publications.

We found these duplicates to be expressed in different ways, as some authors describe them in more detail, and others with just a few words. For example, "documentation of all types is frequently out of date" [13], "outdatedness" [1], "software documentation is rarely, if ever, updated" [8] and "one of the highest costs of maintaining documentation for a large system is to ensure that it is kept in-sync within itself and among its related artifacts, a practice that may require continuous review" [6] are all related with the same concern. So for this step we have considered just one way to express the issue that would make the underlying quality attribute easier to attain;

(3) Define quality attributes that should be considered when writing or maintaining documentation from the identified issues. For this purpose, we have converted the different expressions of issues to quality attributes that can be very useful in a certain categorization or in a future comparison. Some examples of expressions and their associated quality attribute are: "organizations try to re-document their software systems, but this is a costly operation that would benefit from a clear indication of the software documents to focus on" [7] (cost), "many teams either develop too much documentation or too little documentation" [20] (sufficiency) and "lack of documentation" [10] (completeness).

(4) Analyze the identified quality attributes and propose relationships between them, based on the literature and on our own experience.

For example, one work mentioned that "it is very important that the documentation is inexpensive and easy to evolve; otherwise it won't be updated at all, becoming inconsistent, and thus negating the benefits of producing framework documentation" (up-to-dateness and consistency) [2] and other mentioned that "correct documentation provides accurate information in accordance with facts" (correctness and accuracy) [1].

\subsection{Identified Attributes}

From the 14 collected publications we found a total of 91 issues. Many of these were closely related in content, which constituted some sort of duplication.

In order to have all the issues in the same level of knowledge we combined some of them to have less granularity and make them easier to compare and relate. The analysis of the issues led us to identifying the 16 quality attributes that are briefly described below. A summary of which quality attributes is referenced by each publication is given in Table 1 .

- Accessibility. Easy to reach and handle documentation artifacts [7].

- Accuracy. Writing information that it's in conformity with the documentation domain $[1,2]$.

- Automatability. Having programmatic processes when writing or maintaining documentation to avoid manual human work [6].

- Completeness. Containing complete content on documentation provided all necessary details [1, 2, 4, 7, 9, 10, 18, 20].

- Consistency. Maintaining consistent uniform and coherent with the whole document $[2,18]$.

- Correctness. Including precise content into documentation artifacts $[13,18]$.

- Cost. Using an adjusted cost process when writing or maintaining the artifacts $[2,7,13]$.

- Effort. The cognitive load that's necessary to write or maintain documentation [2].

- Readability. The documentation text is clear and legible [6].

- Relevance. Content related to the overall purpose of documentation [2, 20].

- Standardization. Implementing and defining standards and guidelines for teams to follow [2, 7].

- Sufficiency. Providing sufficient documentation to fulfil the goals of the intended reader $[7,9,13,15]$.

- Understandability. The documentation content is comprehensible to intended readers [2, 12].

- Up-to-dateness. Maintaining artifacts updated [1, 6-9, 13, $15,18,20]$.

- Usability. Satisfaction of use of documentation in general $[1,2]$.

- Usefulness. To have utility and positive impact to the endusers [1, 2, 13].

Table 1: Quality attributes mentioned on each publication

\begin{tabular}{|c|c|c|c|c|c|c|c|c|c|c|c|c|c|c|}
\hline Attributes & {$[6]$} & {$[2]$} & {$[7]$} & {$[18]$} & {$[9]$} & {$[12]$} & {$[13]$} & {$[8]$} & {$[20]$} & {$[4]$} & {$[10]$} & {$[1]$} & {$[15]$} & {$[19]$} \\
\hline Accessibility & & & $\checkmark$ & & & & & & & & & & & \\
\hline Accuracy & & $\checkmark$ & & & & & & & & & & $\checkmark$ & & \\
\hline Automatability & $\checkmark$ & & & & & & & & & & & & & \\
\hline Completeness & & $\checkmark$ & $\checkmark$ & $\checkmark$ & $\checkmark$ & & & & $\checkmark$ & $\checkmark$ & $\checkmark$ & $\checkmark$ & & \\
\hline Consistency & & $\checkmark$ & & $\checkmark$ & & & & & & & & & & $\checkmark$ \\
\hline Correctness & & & & $\checkmark$ & & & $\checkmark$ & & & & & & & \\
\hline Cost & & $\checkmark$ & $\checkmark$ & & & & $\checkmark$ & & & & & & & $\checkmark$ \\
\hline Effort & & $\checkmark$ & & & & & & & & & & & & \\
\hline Readability & & & & & & & $\checkmark$ & & & & & $\checkmark$ & $\checkmark$ & $\checkmark$ \\
\hline Relevance & & & & & & & & & & & & & $\checkmark$ & $\checkmark$ \\
\hline Standardization & & $\checkmark$ & $\checkmark$ & & & & & & & & & & & $\checkmark$ \\
\hline Sufficiency & & & $\checkmark$ & & $\checkmark$ & & $\checkmark$ & & & & & & $\checkmark$ & $\checkmark$ \\
\hline Understandability & & $\checkmark$ & & & & $\checkmark$ & & & & & & & & $\checkmark$ \\
\hline Up-to-dateness & $\checkmark$ & & $\checkmark$ & $\checkmark$ & $\checkmark$ & & $\checkmark$ & $\checkmark$ & $\checkmark$ & & & $\checkmark$ & $\checkmark$ & $\checkmark$ \\
\hline Usability & & $\checkmark$ & & & & & & & & & & $\checkmark$ & & $\checkmark$ \\
\hline Usefulness & & $\checkmark$ & & & & & $\checkmark$ & & & & & $\checkmark$ & & $\checkmark$ \\
\hline
\end{tabular}




\subsection{Results}

We find that some attributes are alluded to more often in the literature than others, which indicates a bigger awareness of researchers towards them. As seen in Table 1, the most referenced quality attributes are up-to-dateness (10), completeness (8) and sufficiency (5) while the less referenced are accessibility (1), automatability (1) and effort (1).

We have also analyzed the relationship between the attributes based on the literature and on our own experience. We propose a set of relationships between the quality attributes, which is depicted by Figure 1. We believe this can provide a clearer view of what are the most pressing concerns around software documentation and possibly help to guide finding the most appropriate patterns.

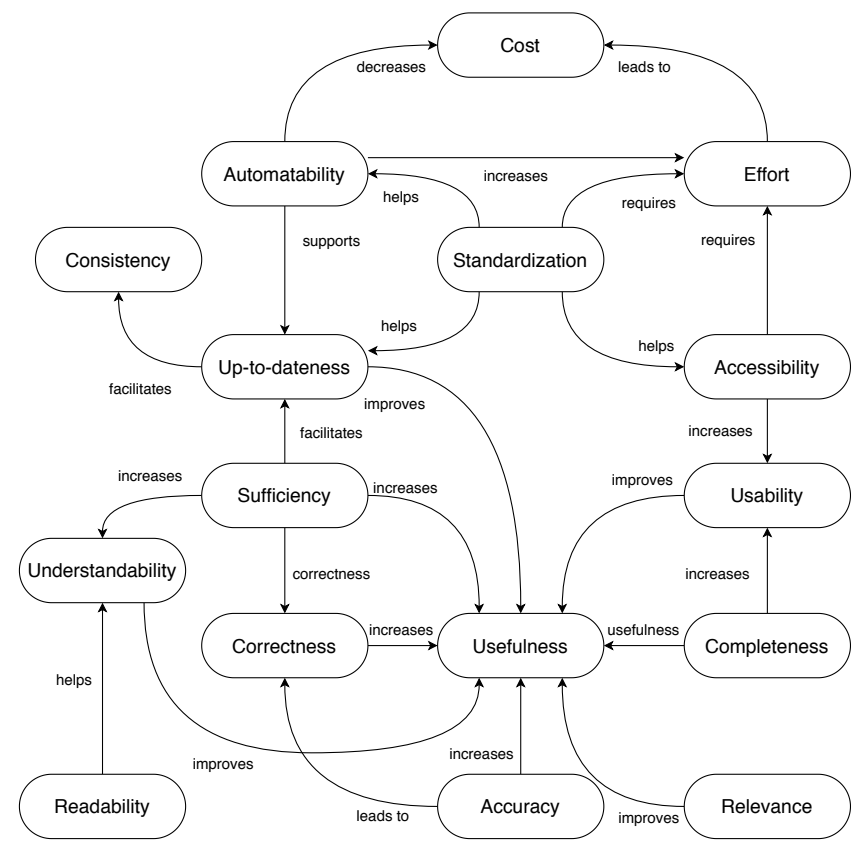

Figure 1: Relationships between quality attributes.

\section{DOCUMENTATION PATTERNS}

This section has the goal of identifying which documentation patterns have been written and what are their constituent parts (e.g., context, problem, solution, forces and main quality attributes that they try to address) to build a catalog of documentation patterns, but also to help identifying differences and commonalities between them, capture these relationships, and support guiding software development teams to in choosing the most appropriate pattern language and pattern for their context.

\subsection{Methodology}

We surveyed the literature for software documentation patterns, and structured our analysis according to the following phases:

(1) Collect works that identify any type of documentation pattern. We searched for literature using Google Scholar using terms related to software documentation such as "patterns software documentation", "software documentation", "software documentation issues" and "software documentation practice". The relevant literature was chosen based on title and abstract;

(2) Describe as patlets all the patterns that have been found, with a focus on the problems and solutions;

(3) Identify also each pattern group and the relationships between patterns that is reported by existing literature;

(4) Combine the gathered patterns into a single pattern map showing the different pattern languages and pattern categories;

(5) Rearrange the patterns to form a new pattern map;

(a) Regroup patterns by purpose;

(b) Analyse the new pattern groups and establish relationships between the patterns within each group that had not yet been identified in the literature;

(c) Merge similar/duplicated patterns that can be merged into one more general pattern that consists of several others;

(d) Identify patterns that fall outside the scope of our analysisin particular, patterns that are not specific to software documentation, or patterns for which we don't have enough information to conclude that they are related to the software documentation.

\subsection{Identified Patterns}

A total of 114 documentation patterns were identified over the 14 publications, 6 of which described patterns $[2,6,10,12,15,19]$. We extracted from this literature several pattern characteristics, such as their problem, solution, pattern category, forces and quality attributes. Some of the patterns don't share the same format, and for some it was not possible to clearly find the context, the problem and the pattern category. We have compiled the available information as a set of patlets, which we make available in Appendix A.

The different pattern languages and categories that we have identified are shown in Figure 2, which depicts 14 different pattern categories and 90 relationships.

\subsection{Results}

The process of regrouping patterns by purpose resulted in the pattern map shown in Figure 3. It helped us to identify 14 different groups, combining patterns from different sources, and within them, 76 relationships between the patterns.

Within these new groups we identified 16 similar patterns that we chose to merge. These are Code-comment Proximity and Embed content in source code; Documentation Portfolio and Documentation Templates; Document Templates and Separation of Contents and Layout; Conceptual writing, Glossary and Project Dictionary; Graded examples and Tutorials information with examples; and finally Framework Overview, Overview Information and The Big Picture.

This new pattern map also shows the 19 patterns that were excluded from our analysis, as we considered them not to be specific to the domain of software documentation and could easily be applicable to other fields. This choice was made merely due to limited time and resources, and we intend to revisit these patterns in future work. 


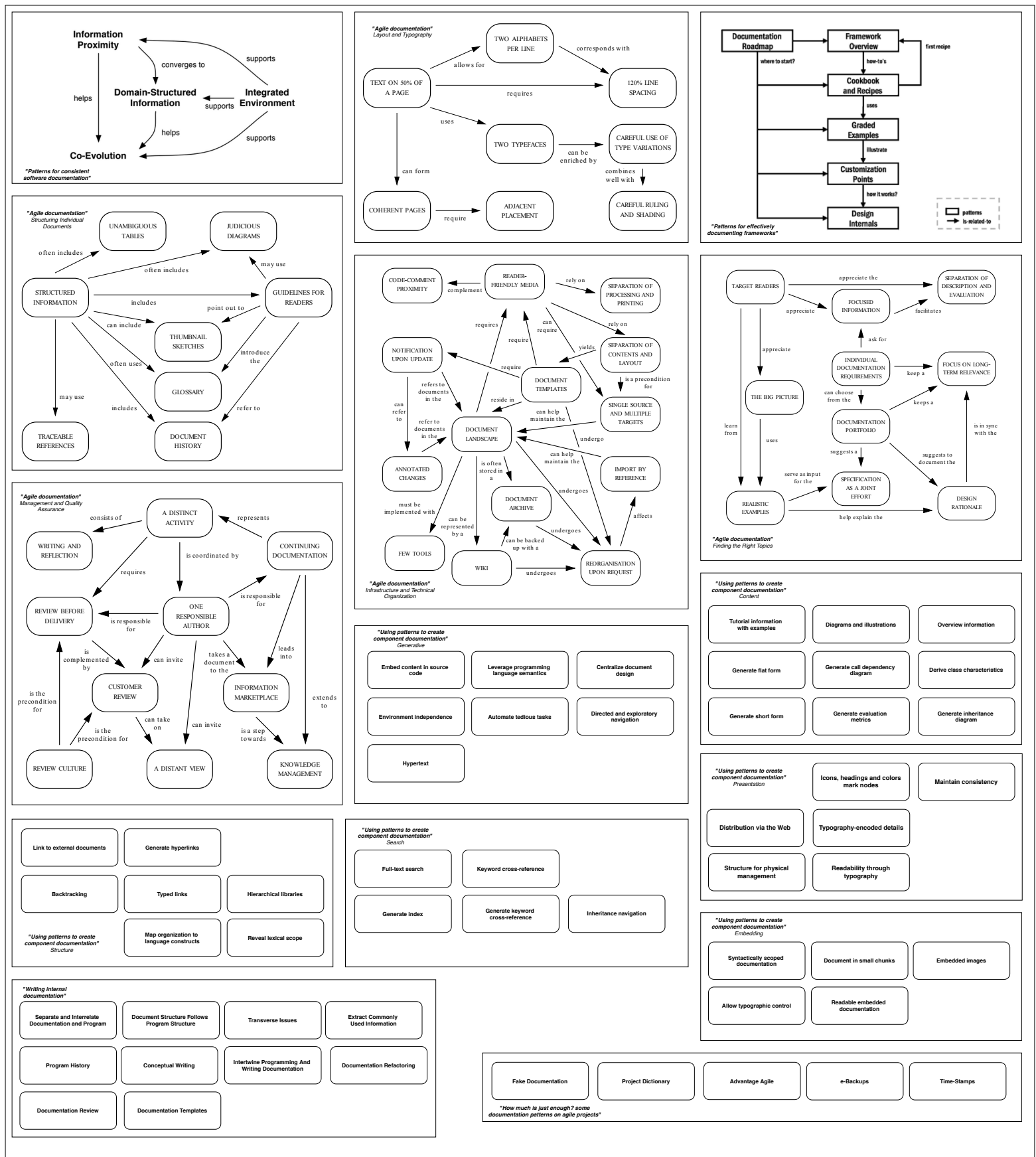

Figure 2: Pattern map that contains information from the extracted literature which describes 90 relationships between patterns among 14 different pattern groups. 


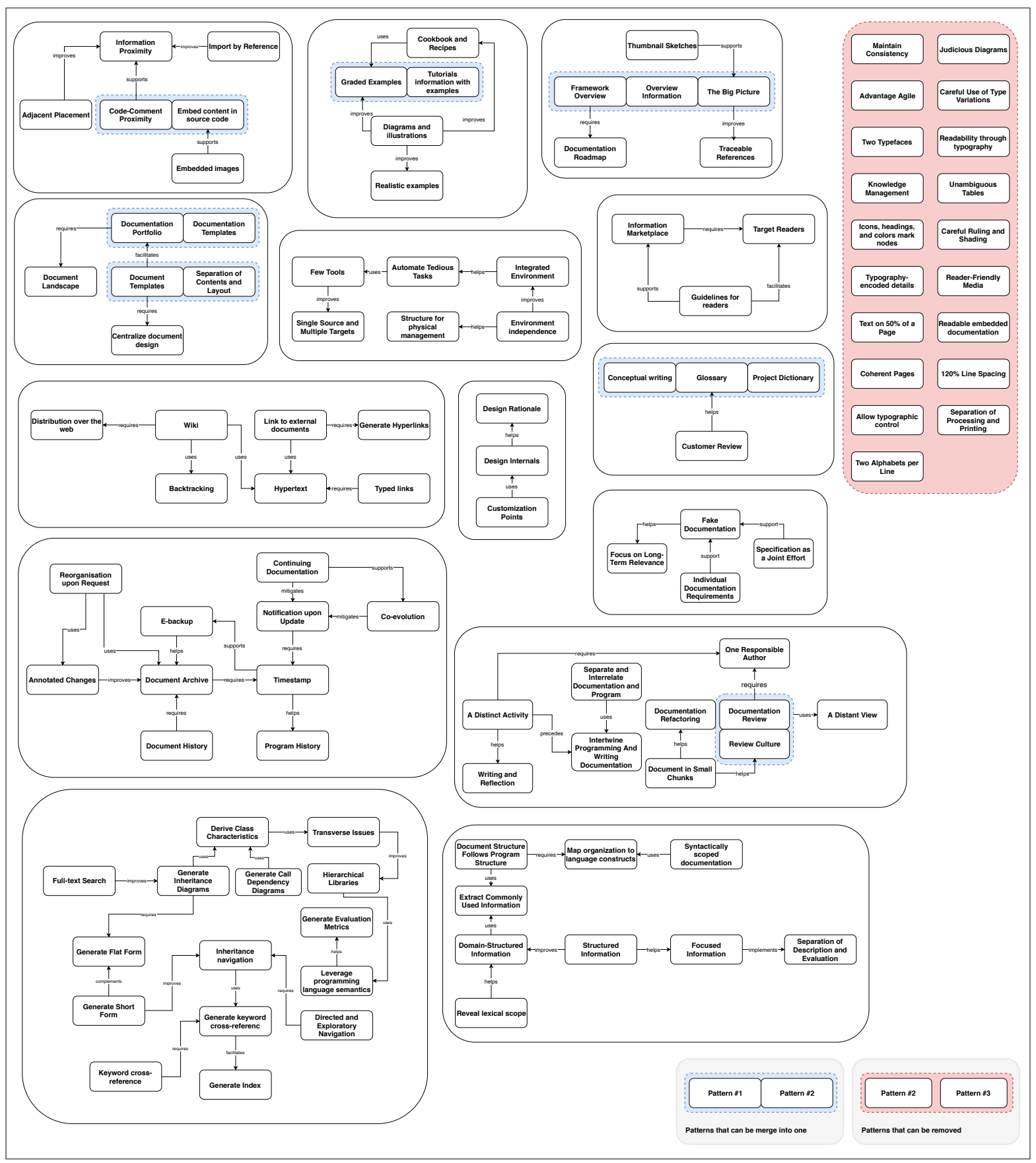

Figure 3: A second pattern map, providing another view over the patterns, and showing 14 rearranged groups of already existing patterns based on each pattern similarity and 76 new identified relationships between patterns. Similar patterns that can be merged into one and potential patterns to remove based on the previously defined criteria were considered as well. 
An analysis of the number of patterns where each quality attribute is a concern rendered the result shown in Table 2. We can conclude that the attributes most addressed are usefulness (38), accessibility (29) and usability (25) and that the least are correctness (2) and accuracy (1).

\begin{tabular}{|c|c|}
\hline $\begin{array}{c}\text { Quality } \\
\text { Attributes }\end{array}$ & $\begin{array}{c}\text { Number of } \\
\text { Related Patterns }\end{array}$ \\
\hline Usefulness & 38 \\
\hline Accessibility & 29 \\
\hline Usability & 25 \\
\hline Consistency & 23 \\
\hline Understandability & 18 \\
\hline Readability & 17 \\
\hline Relevance & 15 \\
\hline Standardization & 11 \\
\hline Automatability & 10 \\
\hline Up-to-dateness & 10 \\
\hline Effort & 6 \\
\hline Cost & 4 \\
\hline Completeness & 4 \\
\hline Sufficiency & 3 \\
\hline Correctness & 2 \\
\hline Accuracy & 1 \\
\hline
\end{tabular}

Table 2: Relationship between quality attributes and number of times they are related with the extracted patterns

\section{CONCLUSIONS}

We analyzed 93 issues from 14 different publications which resulted in a total of 16 quality attributes that are common in the use of software documentation. We also analyzed a total of 114 documentation patterns over 6 publications and their overview provided a concise definition (patlets) that consists of pattern category, solution, problem, forces, related quality attributes and relationship with other patterns.

To answer our first research question ${ }^{1}$ we have identified a set of quality attributes for software documentation by reviewing existing literature (Section 2.2). The answer to our second research question ${ }^{2}$ is supported by the set of patlets included in Appendix A and by the pattern maps depicted by Figures 2 and 3 .

The literature contains several catalogs on specific kinds and aspects of software documentation. They are dispersed and organized in different levels of granularity (regarding different types of documentation and audience). We expect that by providing these new ways to navigate this knowledge will help development teams adopt these practices.

In future work we will use these results as a starting point for an empirical study of how these patterns, pattern relationships and quality attributes influence each other, and hopefully provide further insights on how to improve the adoption of software documentation patterns.

${ }^{1}$ RQ1: What quality attributes are important when creating and using software documentation?

${ }^{2} R Q 2:$ What are the best practices for documenting software?

\section{REFERENCES}

[1] Emad Aghajani, Csaba Nagy, Olga Lucero Vega-Márquez, Mario Linares-Vásquez, Laura Moreno, Gabriele Bavota, and Michele Lanza. 2019. Software Documentation Issues Unveiled. In Proceedings of the 41st International Conference on Software Engineering (ICSE '19). IEEE Press, Piscataway, NJ, USA, 1199-1210. https://doi.org/10.1109/ICSE.2019.00122

[2] Ademar Aguiar and Gabriel David. 2011. Patterns for Effectively Documenting Frameworks. Transactions on pattern languages of programming 2 (2011), 79-124. https://doi.org/10.1007/978-3-642-19432-0_5

[3] Christopher Alexander. 1977. A pattern language: towns, buildings, construction. Oxford university press, Berkeley, USA.

[4] Felix Bachmann, Len Bass, Jeromy Carriere, Paul Clements, David Garlan, James Ivers, Robert Nord, and Reed Little. 2000. Software architecture documentation in practice: Documenting architectural layers. Technical Report. Carnegie-Mellon Univ Pittsburgh Pa Software Engineering Inst.

[5] Frank Buschmann, Regine Meunier, Hans Rohnert, Peter Sommerlad, and Michael Stal. 2008. Pattern-oriented software architecture: a system of patterns. Vol. 1. John Wiley \& Sons, England.

[6] Filipe Figueiredo Correia, Ademar Aguiar, Hugo Sereno Ferreira, and Nuno Flores. 2009. Patterns for consistent software documentation. In Proceedings of the 16th Conference on Pattern Languages of Programs. ACM, New York, USA, 12.

[7] Sergio Cozzetti B. de Souza, Nicolas Anquetil, and Káthia M. de Oliveira. 2005. A Study of the Documentation Essential to Software Maintenance. In Proceedings of the 23rd Annual International Conference on Design of Communication: Documenting \&Amp; Designing for Pervasive Information (SIGDOC '05). ACM, New York, NY, USA, 68-75. https://doi.org/10.1145/1085313.1085331

[8] Andrew Forward and Timothy C Lethbridge. 2002. The relevance of software documentation, tools and technologies: a survey. In Proceedings of the $2002 \mathrm{ACM}$ symposium on Document engineering. ACM, New York, USA, 26-33.

[9] Golara Garousi, Vahid Garousi-Yusifoğlu, Guenther Ruhe, Junji Zhi, Mahmoud Moussavi, and Brian Smith. 2015. Usage and usefulness of technical software documentation: An industrial case study. Information and Software Technology 57 (2015), 664 - 682. https://doi.org/10.1016/j.infsof.2014.08.003

[10] Rashina Hoda, James Noble, and Stuart Marshall. 2010. How much is just enough?: some documentation patterns on Agile projects. In Proceedings of the 15th European Conference on Pattern Languages of Programs. ACM, New York, USA, 13.

[11] Takashi Iba and Taichi Isaku. 2012. Holistic Pattern-Mining Patterns: A Pattern Language for Pattern Mining on a Holistic Approach.

[12] J. Kotula. 1998. Using patterns to create component documentation. IEEE Software 15, 2 (March 1998), 84-92. https://doi.org/10.1109/52.663791

[13] Timothy C Lethbridge, Janice Singer, and Andrew Forward. 2003. How software engineers use documentation: The state of the practice. IEEE software 20, 6 (2003), 35-39.

[14] Peter Naur and Brian Randell. 1969. Software Engineering: Report of a conference sponsored by the NATO Science Committee, Garmisch, Germany, 7th-11th October 1968.

[15] Andreas Rüping. 2005. Agile documentation: a pattern guide to producing lightweight documents for software projects. John Wiley \& Sons, England.

[16] Douglas C. Schmidt, Mohamed Fayad, and Ralph E. Johnson. 1996. Software Patterns. Commun. ACM 39, 10 (Oct. 1996), 37-39. https://doi.org/10.1145/ 236156.236164

[17] Douglas C Schmidt, Michael Stal, Hans Rohnert, and Frank Buschmann. 2013. Pattern-Oriented Software Architecture, Patterns for Concurrent and Networked Objects. Vol. 2. John Wiley \& Sons, England.

[18] G. Uddin and M. P. Robillard. 2015. How API Documentation Fails. IEEE Software 32, 4 (July 2015), 68-75. https://doi.org/10.1109/MS.2014.80

[19] Thomas Vestdam. 2001. Writing Internal Documentation.. In EuroPLoP. ACM, New York, USA, 511-534.

[20] Marcello Visconti and Curtis R Cook. 2002. An overview of industrial software documentation practice. In 12th International Conference of the Chilean Computer Science Society, 2002. Proceedings. IEEE, Chile, 179-186. 


\section{A PATLETS FOR THE IDENTIFIED PATTERNS}

The patterns identified in the literature are specified below as patlets [5]. This format contains the pattern name, the problem, the solution, the quality attributes related to that pattern and the corresponding pattern category (if mentioned in the publication by the authors).

\section{Patterns for Consistent Software Documentation} Information Proximity [6]

Problem: "How to preserve documentation consistency when fragments of related content are scattered across documents?"

Solution: "Keep related information fragments easily accessible from each other, using a single source, links, views, or transclusion, for example, so that it may be easier to assess if they are in-sync."

Quality attributes: Consistency; Up-to-dateness; Readability;

\section{Co-Evolution [6]}

Problem: "When to update a related piece of information in documentation?"

Solution: "When a change is introduced, update the related information parts."

Quality attributes: Up-to-dateness;

\section{DOMAIN-STRUCTURED INFORMATION [6]}

Problem: "How to structure the information in documentation?"

Solution: "Organize contents according to their domain, so that the information form directly relates to domain concepts."

Quality attributes: Relevance; Usefulness;

INTEGRATE D ENVIRONMENT [6]

Problem: "How to support the maintenance of consistency between independent artifacts with related content?"

Solution: "Use an integrated environment, where several types of artifact and their relations may be maintained uniformly."

Quality attributes: Consistency;

Patterns for Effectively Documenting Frameworks
Documentation RoADMAP [2]

Problem: "How to help readers on quickly finding in the overall documentation their way to the information they need?"

Solution: "Start by providing a roadmap for the overall documentation, one that reveals its organization, how the pieces of information fit together, and that elucidates readers of different audiences about the main entry points and the paths in the documentation that may drive them quickly to the information they are looking for, especially at their first contact."

Quality attributes: Accessibility; Usefulness;

Framework Overview [2]

Problem: "How to help readers on getting a quick, but precise, first impression of a new framework?"

Solution: "Provide an introductory document, in the form of a framework overview, that describes the domain covered by the framework in a clear way, i.e. the application domain and the range of solutions for which the framework was designed and is applicable."

Quality attributes: Accessibility; Relevance; Understandability; Usefulness;

\section{COоквоOK AND RecIPEs [2]}

Problem: "How to quickly provide users with information that helps them learning how to use the framework?"

Solution: "Provide a collection of recipes, one for each framework customization, organized in a cookbook, which acts as a guide to the contents of all its recipes."

Quality attributes: Usability; Usefulness;

Graded EXAMPLES [2]

Problem: "How to help readers on getting started fast using a framework both for simple and complex kinds of usage?"

Solution: "Provide a small but representative graded set of training examples to illustrate the framework's applicability and features, each one illustrating a single new way of customization, smoothly growing in complexity, and eventually altogether providing complete coverage."

Quality attributes: Relevance; Understandability; Usefulness; Customization Points [2]

Problem: "How to help readers learn in detail how to customize a specific part of a framework?"

Solution: "Provide a list of the framework's customization points, also known as hot-spots, i.e., the points of predefined refinement where framework customization is supported, and, for each one, describe in detail the hooks it provides and the hot-spot subsystem that implements its flexibility."

Quality attributes: Relevance; Understandability; Usefulness; DESIGN INTERNALS [2]

Problem: "How to help framework users on quickly grasping the design and implementation of a framework to support them on achieving advanced customizations, not typical, or not specifically documented?"

Solution: "Provide concise detailed information about the design internals of the framework, especially the areas designed to support configuration, known as hot-spots. You should start by describing how the framework hot-spots support configuration."

Quality attributes: Understandability; Usability; Usefulness;

Using Patterns To Create Component Documentation ENVIRONMENT INDE PENDENCE [12]

Solution: "The documentation system must be feasible and effective in any operating environment."

Pattern category: "Generative Patterns"

Quality attributes: Accuracy;

\section{HyPertext [12]}

Solution: "Present the documentation as hypertext to enable effective navigation of the information."

Pattern category: "Generative Patterns"

Quality attributes: Accessibility; Usability;

EMBED CONTENT IN SOURCE CODE [12]

Solution: "Ensure that the component documentation is synchronized with the component source code."

Pattern category: "Generative Patterns"

Quality attributes: Up-to-dateness; 


\section{Automate tedious tasks [12]}

Solution: "Automate as many documentation production and maintenance tasks as possible to relieve the workload of the authors."

Pattern category: "Generative Patterns"

Quality attributes: Automatability;

LEVERAge PROgRAMming LANGUAGE SEMANTICS [12]

Solution: "Take advantage of all the information about the component that is inherent in the source code."

Pattern category: "Generative Patterns"

Quality attributes: Usefulness;

\section{Centralize document design [12]}

Solution: "Centralize the layout and design of the documentation to ensure its consistency."

Pattern category: "Generative Patterns"

Quality attributes: Consistency; Usability;

DIRECTED AND EXPLORATORY NAVIGATION [12]

Solution: "Provide a means for both directed and exploratory navigation to support reader education and research."

Pattern category: "Generative Patterns"

Quality attributes: Accessibility; Usability;

TUTORIAL INFORMATION WITH EXAMPLES [12]

Solution: "Provide the client with information on how to use the component in specific, common use cases."

Pattern category: "Content Patterns"

Quality attributes: Usability; Usefulness;

Diagrams and ILlustrations [12]

Solution: "Include diagrams and illustrations in documentation for clarification purposes"

Pattern category: "Content Patterns"

Quality attributes: Readability; Usability; Usefulness;

OVERVIEW INFORMATION [12]

Solution: "Provide high-level overview descriptions and summaries in the documentation for each component, library, and class."

Pattern category: "Content Patterns"

Quality attributes: Relevance; Understandability; Usefulness;

GENERATE SHORT FORM [12]

Solution: "Automatically create a brief summary of the extensions that a derived class makes to its base class."

Pattern category: "Content Patterns"

Quality attributes: Understandability; Usefulness;

GENERATE FLAT FORM [12]

Solution: "Automatically create a complete summary of all class members from all parent classes."

Pattern category: "Content Patterns"

Quality attributes: Understandability; Usefulness;

GENERATE INHERITANCE DIAGRAMS [12]

Solution: "Automatically generate diagrams depicting the inheritance structure of the classes."

Pattern category: "Content Patterns"

Quality attributes: Automatability;
GENERATE CALL DEPENDENCY DIAGRAMS [12]

Solution: "Automatically generate diagrams depicting the interclass dependencies in each library or component."

Pattern category: "Content Patterns"

Quality attributes: Readability; Usability; Usefulness;

GENERATE EVALUATION METRICS [12]

Solution: "Automatically generate object-oriented code metrics to help clients evaluate the components."

Pattern category: "Content Patterns"

Quality attributes: Automatability; Usefulness;

DERIVE CLASS CHARACTERISTICS [12]

Solution: "Automatically list the descriptive attributes that characterize each class."

Pattern category: "Content Patterns"

Quality attributes: Automatability; Usefulness;

MAP ORGANIZATION TO LANGUAGE CONSTRUCTS [12]

Solution: "Structure the hypertext nodes and the links between them to parallel the programming language constructs used to implement the component."

Pattern category: "Structure Patterns"

Quality attributes: Correctness; Relevance;

\section{HiERARCHICAL LIBRARIES [12]}

Solution: "In the documentation, provide a mechanism for grouping related classes together to form subsystem libraries."

Pattern category: "Structure Patterns"

Quality attributes: Consistency; Readability;

LINK TO EXTERNAL DOCUMENTS [12]

Solution: "Join supplementary documents to the component documentation through hypertext links"

Pattern category: "Structure Patterns"

Quality attributes: Accessibility;

\section{BACKTRACKING [12]}

Solution: "Provide the user with links that backtrack to related, higher levels of the documentation"

Pattern category: "Structure Patterns"

Quality attributes: Accessibility; Usability;

GENERATE HYPERLINKS [12]

Solution: "Automatically hyperlink each occurrence of a software artifact's name to its full documentation."

Pattern category: "Structure Patterns"

Quality attributes: Accessibility; Automatability;

\section{REVEAL LEXICAL SCOPE [12]}

Solution: "Structure the documentation to reflect the lexical scope of the software artifacts within a component."

Pattern category: "Structure Patterns"

Quality attributes: Consistency; Usability;

TYPED LiNks [12]

Solution: "Classify hyperlinks by type to enable more directed, selective navigation through the component documentation."

Pattern category: "Structure Patterns"

Quality attributes: Accessibility; Usefulness; 


\section{KEYWORD CROSS-REFERENCE [12]}

Solution: "Provide a mechanism to help clients find classes in a component within categories of interest."

Pattern category: "Search Patterns"

Quality attributes: Accessibility; Usability;

INHERITANCE NAVIGATION [12]

Solution: "Provide an efficient, convenient means to quickly navigate through the inheritance structure for a given class."

Pattern category: "Search Patterns"

Quality attributes: Accessibility; Usability;

\section{FULL-TEXT SEARCH [12]}

Solution: "Provide utilities to search through the full text of the documentation."

Pattern category: "Search Patterns"

Quality attributes: Accessibility; Usability;

\section{GENERATE INDEX [12]}

Solution: "Automatically determine which words and phrases in the component documentation are most significant and generate an index from them."

Pattern category: "Search Patterns"

Quality attributes: Automatability; Usefulness;

GENERATE KEYWORD CROSS-REFERENCE [12]

Solution: "Automatically parse class names to create a minimal list of cross-reference keywords."

Pattern category: "Search Patterns"

Quality attributes: Automatability; Usefulness; P39

\section{Maintain CONSISTENCY [12]}

Solution: "Make the visual appearance of the documentation consistent."

Pattern category: "Presentation Patterns"

Quality attributes: Consistency; Usability;

Distribution via the WeB [12]

Solution: "Use Web pages on the Internet to provide access to the most current version of the documentation."

Pattern category: "Presentation Patterns"

Quality attributes: Accessibility; Usability;

ICONS, HEADINGS, AND COLORS MARK NODES [12]

Solution: "Use pictorial icons, standard headings, and/or colors to mark the context and purpose of hypertext nodes in the documentation."

Pattern category: "Presentation Patterns"

Quality attributes: Readability; Usability;

\section{READABILITY THROUGH TYPOGRAPHY [12]}

Solution: "Graphically represent the textual information clearly and effectively."

Pattern category: "Presentation Patterns"

Quality attributes: Readability; Usability;

TYPOGRAPHY-ENCODED DETAILS [12]

Solution: "Use typographic conventions to encode syntactic details where programming language syntax is directly included in the documentation."

Pattern category: "Presentation Patterns"
Quality attributes: Readability; Usability; STRUCTURE FOR PHYSICAL MANAGEMENT [12]

Solution: "Design the physical repository of the documentation to enable its easy versioning and administration as a unit."

Pattern category: "Presentation Patterns"

Quality attributes: Up-to-dateness; Usefulness; SYNTACTICALLY SCOPED DOCUMENTATION [12]

Solution: "Use syntactic rules to associate embedded documentation with the programming-language construct it documents."

Pattern category: "Embedding Patterns"

Quality attributes: Consistency; Usefulness;

Document IN SMALl CHUNKS [12]

Solution: "Structure the embedded comment text so that software artifacts are documented in small increments."

Pattern category: "Embedding Patterns"

Quality attributes: Consistency; Usefulness;

Allow typographic control [12]

Solution: "Program the extraction tool to support typographic markup within the manually authored documentation text."

Pattern category: "Embedding Patterns"

Quality attributes: Automatability;

READABLE EMBEDDED DOCUMENTATION [12]

Solution: "Design a documentation-embedding syntax that enhances the readability of the source code."

Pattern category: "Embedding Patterns"

Quality attributes: Readability; Usefulness;

EMBEDDED IMAGES [12]

Solution: "Centralize file management by embedding ASCIIencoded images within the source code for later extraction, decoding, and inclusion in the final documentation."

Pattern category: "Embedding Patterns"

Quality attributes: Standardization;

How Much is Just Enough? Some Documentation Patterns on Agile Projects

Fake Documentation [10]

Problem: "How do you co-ordinate the timing of documentations produced across Agile and non-Agile projects?"

Solution: "Time the production of a minimal amount of traditional documentation to co-ordinate with non-Agile teams."

Quality attributes: Cost; Effort; Sufficiency;

Time STamp [10]

Problem: "How do you clarify who initiated a change request?"

Solution: "Document change decisions with timestamp on a wiki."

Quality attributes: Standardization; Up-to-dateness;

$$
\text { E-BACKUP [10] }
$$

Problem: "How do you avoid losing all your story cards and task post-its?"

Solution: "Make electronic backups of paper artifacts."

Quality attributes: Cost; Effort; Standardization; 


\section{Project Dictionary [10]}

Problem: "How do you translate customer's business requirements into technical tasks?"

Solution: "Engage your customers to document business terms, their meanings, and contexts of use into a project dictionary."

Quality attributes: Consistency; Correctness; Understandability;

\section{Advantage Agile [10]}

Problem: "How do you demonstrate the Agile advantage over successful non-Agile projects measured using traditional metrics?"

Solution: "Document positive customer feedback."

Quality attributes: Usefulness;

\section{Agile Documentation - A Pattern Guide to Producing Lightweight Documents for Software Projects}

\section{TARget READERS [15]}

Problem: "How can the project team ensure that the documents they produce will be appreciated?"

Solution: "First and foremost, each document must have a target readership, and must address these readers in order to prove useful."

Pattern category: "Finding the Right Topics"

Quality attributes: Relevance; Usefulness;

\section{FOCUSED INFORMATION [15]}

Problem: "How can documents be prevented from meandering and getting nowhere fast?"

Solution: "A clear and identifiable focus on a particular topic makes a document concise and straightforward. The straightforward document offers the information relevant to this topic, but no more than that."

Pattern category: "Finding the Right Topics"

Quality attributes: Consistency; Relevance;

\section{Individual Documentation ReQUiRements [15]}

Problem: "How can unnecessary documentation requirements be avoided?"

Solution: "The most effective approach towards documentation is for each project to define its documentation requirements individually."

Pattern category: "Finding the Right Topics"

Quality attributes: Sufficiency;

\section{Documentation Portfolio [15]}

Problem: "How can teams reuse the knowledge about which documents might be required in their projects?"

Solution: "A documentation portfolio describes which documents might be necessary in a software project, and their scope. If an organisation sets up such a portfolio, projects can choose those documents they need, checking the necessity of each candidate document individually."

Pattern category: "Finding the Right Topics"

Quality attributes: Cost; Effort; Standardization;

Focus on Long-Term Relevance [15]

Problem: "How can projects avoid producing documentation that expires too soon?"
Solution: "There is much value in documentation that focuses on issues with a long-term relevance - issues that will play a role in a later project phase or in future projects."

Pattern category: "Finding the Right Topics"

Quality attributes: Up-to-dateness;

\section{SPECIFICATION AS A JoInt EFfort [15]}

Problem: "How can development projects ensure that they head in the direction the customer wants?"

Solution: "Every development project requires a specification, which reflects the requirement analysis done jointly by the project team and the customer."

Pattern category: "Finding the Right Topics"

Quality attributes: Relevance; Usefulness;

Design Rationale [15]

Problem: "How can the team make sure that the foundations are laid for future design changes?"

Solution: "Design documents become a valuable source of information if they aren't restricted to describing the actual design, but also focus on the rationale behind the design and explain why the particular design was chosen."

Pattern category: "Finding the Right Topics"

Quality attributes: Completeness;

\section{The Big Picture [15]}

Problem: "How can people be introduced to a project without being confronted with a deluge of technical details?"

Solution: "A good feel for a project is best conveyed through a description of the 'big picture' of the architecture that underlies the system under construction."

Pattern category: "Finding the Right Topics"

Quality attributes: Accessibility; Relevance; Understandability; Usefulness;

Separation of Description and Evaluation [15]

Problem: "How can authors prevent loss of credibility?"

Solution: "Authors gain credibility if, in their documents, they clearly separate description from evaluation."

Pattern category: "Finding the Right Topics"

Quality attributes: Consistency;

\section{REALISTIC Examples [15]}

Problem: "How can abstract material be explained in a comprehensible way?"

Solution: "Project documents are much more convincing if they include realistic examples from the project's context."

Pattern category: "Finding the Right Topics"

Quality attributes: Accessibility; Completeness; Understandability;

\section{STRUCTURED INFORMATION [15]}

Problem: "How can information be presented in an easily accessible way?"

Solution: "Most project documents are best organised as sequential yet well-structured text. This begins with well-chosen chapters and sections, but may well extend to using textual building blocks consistently throughout a document."

Pattern category: "Structuring Individual Documents"

Quality attributes: Accessibility; Consistency; 


\section{Judicious Diagrams [15]}

Problem: "How can authors provide an overview of structures and processes in a convenient way?"

Solution: "Diagrams can provide excellent overviews, while an accompanying text explains details to the extent that is necessary."

Pattern category: "Structuring Individual Documents"

Quality attributes: Completeness; Understandability;

UNAmbiguOUS TABLES [15]

Problem: "How can authors present systematic information in a precise way?"

Solution: "Tables offer a compact format for the concise and unambiguous presentation of information."

Pattern category: "Structuring Individual Documents"

Quality attributes: Consistency; Relevance;

GUIDELINES FOR READERS [15]

Problem: "How can potential readers be informed whether they should read a document, and if so, on which parts they should focus?"

Solution: "Some brief guidelines at the beginning of each document can inform potential readers of the purpose the document serves and explain how different groups of readers should approach the document."

Pattern category: "Structuring Individual Documents"

Quality attributes: Accessibility; Understandability;

Thumbnail Sketches [15]

Problem: "How can readers get an overview of the topics dealt with in a document?"

Solution: "Thumbnail sketches provide brief descriptions of the sections of a document, including the section's general goals, as well as its major ideas."

Pattern category: "Structuring Individual Documents"

Quality attributes: Relevance; Understandability;

\section{Traceable References [15]}

Problem: "How can documents be linked to each other?"

Solution: "A document should include references to other documents only if readers can obtain those documents without much effort."

Pattern category: "Structuring Individual Documents"

Quality attributes: Accessibility; Consistency;

\section{GLOSSARY [15]}

Problem: "How can authors make sure that readers understand the vocabulary used in a document?"

Solution: "A glossary can explain technical terms as well as the terms specific to the application domain."

Pattern category: "Structuring Individual Documents"

Quality attributes: Readability; Standardization; Understandability;

\section{Document History [15]}

Problem: "How can confusion be avoided between versions of a document?"

Solution: "A document history can explain the differences to previous versions of a document, and can relate the document to versions of the software it describes."

Pattern category: "Structuring Individual Documents"
Quality attributes: Up-to-dateness;

Text on 50\% of a Page [15]

Problem: "How much space on a page should be devoted to text?"

Solution: "About $50 \%$ of the page should be devoted to text."

Pattern category: "Layout and Typography"

Quality attributes: Accessibility; Readability; Usability;

Two Alphabets per Line [15]

Problem: "What is the optimum line width?"

Solution: "Approximately two lowercase alphabets of the standard typeface should fit on one line."

Pattern category: "Layout and Typography"

Quality attributes: Accessibility; Readability; $120 \%$ Line SPacing [15]

Problem: "What is the optimum line spacing?"

Solution: "The best line spacing is roughly $120 \%$ of the type size."

Pattern category: "Layout and Typography"

Quality attributes: Accessibility; Readability;

Two Typefaces [15]

Problem: "How many typefaces are appropriate, and which?"

Solution: "In most cases, two typefaces per document are appropriate - a serif typeface for the body text and a sans-serif typeface for the headings."

Pattern category: "Layout and Typography"

Quality attributes: Accessibility; Readability; Usability;

Careful Use of Type Variations [15]

Problem: "How can parts of a text be emphasised?"

Solution: "Type variations can be used for emphasis, but they should be used with care."

Pattern category: "Layout and Typography"

Quality attributes: Accessibility; Readability; Usability; Careful Ruling and Shading [15]

Problem: "How can table cells be separated?"

Solution: "Careful ruling and shading leads to highly legible tables."

Pattern category: "Layout and Typography"

Quality attributes: Accessibility; Readability; Usability;

Adjacent Placement [15]

Problem: "How can tables and diagrams be integrated into text?"

Solution: "Diagrams and tables are best placed close to the text from which they are referenced."

Pattern category: "Layout and Typography"

Quality attributes: Accessibility; Usability;

Coherent Pages [15]

Problem: "What options exist to avoid awkward pagination that tears related information apart?"

Solution: "The reading flow is supported by coherent pages pages that make sure a minimum of related information appears on either side of a page break."

Pattern category: "Layout and Typography"

Quality attributes: Accessibility; Readability; 


\section{Document LANDSCAPE [15]}

Problem: "How can team members get a good overview of what documentation exists in a project?"

Solution: "The project documentation can be represented as a kind of landscape that team members can use as a mental map when they retrieve or add information. A document landscape that roughly forms a tree suits human intuition best."

Pattern category: "Infrastructure and Technical Organization" Quality attributes: Accessibility; Usability; Usefulness;

\section{Document Archive [15]}

Problem: "How can projects avoid the loss of any document versions?"

Solution: "Archiving project documentation offers the advantage that versions can be retrieved when necessary."

Pattern category: "Infrastructure and Technical Organization" Quality attributes: Up-to-dateness;

\section{WIKI [15]}

Problem: "How can documentation be given a more interactive edge?"

Solution: "A Wiki offers access to the project documentation via an intranet server, and in addition allows the team to post notes and messages to others as necessary."

Pattern category: "Infrastructure and Technical Organization" Quality attributes: Accessibility; Usefulness;

\section{Code-Comment Proximity [15]}

Problem: "What is an easy way to maintain documentation that refers to the actual code?"

Solution: "Documentation of the code, to the extent that a project team considers it necessary, is best done through source code comments. Separate documents should be reserved for higher-level issues such as overviews, requirements, design and architecture."

Pattern category: "Infrastructure and Technical Organization" Quality attributes: Consistency; Standardization;

\section{ReAder-Friendly Media [15]}

Problem: "Which is more appropriate: documents intended for on-line use, or documents intended for print?"

Solution: "The choice of a medium must reflect a document's typical usage. The rule of thumb is: print is good for reading, on-line is good for looking things up."

Pattern category: "Infrastructure and Technical Organization"

Quality attributes: Usability;

\section{Separation of Contents and Layout [15]}

Problem: "How can the layouts of text documents be changed and reused easily?"

Solution: "Layout styles can be defined and assigned to content portions. These layout styles can easily be changed and can be reused across documents."

Pattern category: "Infrastructure and Technical Organization"

Quality attributes: Consistency;

\section{Single Source and Multiple Targets [15]}

Problem: "How can multiple views of a document be created without doubling maintenance?"

Solution: "The documentation infrastructure can employ mechanisms that take source documents and automatically generate additional views. Such mechanisms avoid double maintenance and ensure consistency."

Pattern category: "Infrastructure and Technical Organization" Quality attributes: Automatability;

\section{IMPORT BY REFERENCE [15]}

Problem: "How can different documents use the same diagram or table consistently?"

Solution: "Artefacts that need to appear in multiple contexts can be imported by reference into the documents that include them."

Pattern category: "Infrastructure and Technical Organization" Quality attributes: Consistency;

\section{Separation of Processing and Printing [15]}

Problem: "How can projects produce useful, printable documents?"

Solution: "If a team chooses to deliver the project documentation in a print format that is widely available, all readers are able to print the documents, independent of the platform they use."

Pattern category: "Infrastructure and Technical Organization"

Quality attributes: Accessibility; Usefulness;

Document Templates [15]

Problem: "How can all project documents acquire a reasonable structure and a good layout at little cost?"

Solution: "Document templates, once they have been properly designed, impose their structure and layout on all documents that are produced using them."

Pattern category: "Infrastructure and Technical Organization" Quality attributes: Consistency;

Few Tools [15]

Problem: "How can projects minimise the effort spent on the introduction and use of documentation tools?"

Solution: "Almost all projects can manage with a small set of documentation tools."

Pattern category: "Infrastructure and Technical Organization" Quality attributes: Effort;

Annotated Changes [15]

Problem: "How can authors avoid confusion over changes they have made?"

Solution: "While a document is under development, authors can use automatic annotations to identify those parts of the document that have changed recently."

Pattern category: "Infrastructure and Technical Organization"

Quality attributes: Automatability;

NOTIFICATION UPON UPDATE [15]

Problem: "How can readers be prevented from using outdated versions?"

Solution: "Whenever there is a significant change in a project document, all potential readers should be notified of the new version. The notification should roughly explain what has been changed, but should not include the updated material itself."

Pattern category: "Infrastructure and Technical Organization" Quality attributes: Up-to-dateness; 


\section{REORgANISATION UPON REQUEST [15]}

Problem: "How can the documentation infrastructure be maintained?"

Solution: "Frequent reorganisation makes things worse, not better. Reorganisation of the documentation infrastructure should take place only when it is requested by the members of the project team."

Pattern category: "Infrastructure and Technical Organization" Quality attributes: Consistency; Standardization;

\section{A Distinct Activity [15]}

Problem: "How should resources be assigned to documentation activities?"

Solution: "When documentation is considered a distinct project activity, and not just the by-product of coding, it can be assigned its own budget, priority and schedule. Documentation can then be weighed against other project activities."

Pattern category: "Management and Quality Assurance" Quality attributes: Cost;

\section{One Responsible Author [15]}

Problem: "How many people should be responsible for a document?"

Solution: "For each project document, there must be one person who accepts responsibility for it. This person need not write the document alone, but must coordinate the contributions from other people."

Pattern category: "Management and Quality Assurance"

Quality attributes: Standardization;

\section{Continuing Documentation [15]}

Problem: "When should project documentation be written?"

Solution: "Project documentation, when it evolves continuously as the project goes on, offers the advantage that it reflects the last stable state of the project."

Pattern category: "Management and Quality Assurance"

Quality attributes: Effort;

\section{WRITING AND REFLECTION [15]}

Problem: "How can documentation and other project activities stimulate each other?"

Solution: "To get the best out of documentation, team members have to spend time on the actual writing, as well as in reflection on what they have written, preferably in an undisturbed environment."

Pattern category: "Management and Quality Assurance"

Quality attributes: Effort; Standardization;

\section{Review Culture [15]}

Problem: "How can the quality of the project documents be improved?"

Solution: "Documentation can profit a lot from reviews, provided a review culture has been established in which both authors and reviewers feel comfortable."

Pattern category: "Management and Quality Assurance"

Quality attributes: Usefulness;

ReView Before Delivery [15]

Problem: "How can authors receive the right feedback at the right time?"
Solution: "Early reviews are fine as they help the author shape the scope and the structure of a document. But before a document is officially distributed, or delivered to the customer, a review is mandatory."

Pattern category: "Management and Quality Assurance"

Quality attributes: Standardization;

\section{Customer Review [15]}

Problem: "How can a team use documentation to increase customer involvement?"

Solution: "Customer reviews can improve the quality of a document, especially as far as the domain expertise is concerned, and at the same time add to team building and integration."

Pattern category: "Management and Quality Assurance"

Quality attributes: Usefulness; Completeness;

\section{A Distant View [15]}

Problem: "How can authors obtain unbiased feedback?"

Solution: "Authors can obtain unbiased feedback from reviewers who are interested in the topic and who are generally knowledgeable in the field, but who are not involved in the actual work described in the document."

Pattern category: "Management and Quality Assurance"

Quality attributes: Usefulness;

\section{INFORMATION MARKETPLACE [15]}

Problem: "How can good documents be prevented from going sadly unnoticed?"

Solution: "Documents gain more attention if the intended readers are actively invited to read them."

Pattern category: "Management and Quality Assurance"

Quality attributes: Readability; Relevance; Usefulness;

\section{KnOWledge Management [15]}

Problem: "How can future projects profit from a successful project?"

Solution: "Only when project documentation is made available organisation-wide do future projects have a chance of drawing on the expertise gained."

Pattern category: "Management and Quality Assurance"

Quality attributes: Accessibility;

\begin{tabular}{c} 
Writing Internal Documentation \\
\hline Se Parate and Interrelate Documentation AND \\
Program [19] \\
Problem: "A pile of documentation in one hand and a pile of
\end{tabular}
code in the other - how can one find the documentation relevant for a given part of the code?"

Solution: "Separate documentation and program, and use typed links to provide selective and mutual navigation between documentation and program."

Pattern category: "Structural patterns"

Quality attributes: Consistency; Relevance;

Document Structure Follows Program Structure [19]

Problem: "Getting started on writing documentation as well as structuring the documentation is not easy, especially for the inexperienced documentation writer - how can one get started writing and structuring documentation?" 
Solution: "Let the documentation structure follow the program structure when presenting static program structure and program entities."

Pattern category: "Structural patterns"

Quality attributes: Understandability; Usefulness;

Transverse Issues [19]

Problem: "It is not feasible to document all aspects of a program, but which aspects should at least be considered?"

Solution: "Describe and explain program relationships such as collaborating program entities or code dependencies. Relate such transverse issues to relevant program fragments."

Pattern category: "Structural patterns"

Quality attributes: Understandability;

EXTract COMmonly USED Information [19]

Problem: "The documentation often becomes a mess because explanations of program entities become long and complex. It becomes difficult to find relevant explanations, and consequently difficult to update - how can the documentation become more flexible?

Solution: "Different aspects of a program entity should be explained separately, if these aspects are important in more than one context."

Pattern category: "Structural patterns"

Quality attributes: Sufficiency; Usefulness;

Program History [19]

Problem: "Often programmers inadvertently try solutions that have already been proven useless because they have forgotten previous encountered problems - how can this be avoided?"

Solution: "Record the choices made during program development in the documentation. This includes relevant solutions that have been tried but proven useless. In addition, consider recording relevant alternatives such as solutions that are more flexible, optimal or even more aesthetic than the chosen solution."

Pattern category: "Structural patterns"

Quality attributes: Relevance; Understandability; Usefulness; Conceptual Writing [19]

Problem: "Different programmers often use the same words for different things and different words for the same thing - how can we avoid that the documentation inherits such inconsistencies? "

Solution: "Special terminology used about the program must be defined in a central part of the documentation."

Pattern category: "Structural patterns"

Quality attributes: Consistency; Understandability;

\section{Intertwine Programming And Writing}

Documentation [19]

Problem: "Documentation must be written, but when is it time to write it?"

Solution: "Document the overall code design before coding, and document the design details after the coding. In addition, maintain relevant issues that arise during coding by alternating between writing code and documentation."

Pattern category: "Temporal patterns"

Quality attributes: Usefulness;

\section{DOCUMENTATION REFACTORING [19]}

Problem: "The program evolves, and documentation gradually falls behind. Keeping the documentation up-to-date according to every change is simply not feasible - when is it time to update the documentation and how can this be done without constantly changing the entire documentation?"

Solution: "Refactor the documentation on a regular basis either by 1) providing updates to the documentation based on a hot list of changes that need immediate propagation, or 2)revise the entire documentation."

Pattern category: "Maintenance patterns"

Quality attributes: Up-to-dateness;

Documentation Review [19]

Problem: "Documentation varies from programmer to programmer in terms of quality and quantity - how can the overall quality of documentation be evaluated and ensured during coding?"

Solution: "The documentation should be reviewed regularly in order to ensure the adequacy of the documentation. Documentation reviews can for example be performed in connection with code reviews."

Pattern category: "Maintenance patterns"

Quality attributes: Consistency;

Documentation Templates [19]

Problem: "Different programmers have different styles when writing and structuring documentation. Depending on which programmer wrote what in the documentation, some aspects of the program are well explained whereas others are not - how can we ensure some kind of uniformity of the documentation?"

Solution: "Identify overall aspects that should (or can) be addressed in the documentation and create templates for these."

Pattern category: "Stylistic patterns"

Quality attributes: Consistency; Standardization; 